\title{
Effects of Administering Sodium Selenite, Methylseleninic Acid, and Seleno-L-methionine on Glucose Tolerance in a Streptozotocin/ Nicotinamide-Induced Diabetic Mouse Model
}

\author{
Hitoshi Ueno, ${ }^{* a}$ Ryo Shimizu, ${ }^{b}$ Tomofumi Okuno, ${ }^{a}$ Hirofumi Ogino, ${ }^{a}$ Tomohiro Arakawa, ${ }^{a}$ \\ Fumitoshi Sakazaki, ${ }^{c}$ and Katsuhiko Nakamuro ${ }^{d}$ \\ ${ }^{a}$ Faculty of Pharmaceutical Sciences, Setsunan University; 45-1 Nagaotoge-cho, Hirakata, Osaka 573-0101, Japan: \\ ${ }^{b}$ Faculty of Pharmaceutical Sciences, Hiroshima International University; 5-1-1 Hirokoshingai, Kure, Hiroshima \\ 737-0112, Japan: ${ }^{c}$ Faculty of Pharmacy, Osaka Ohtani University; 3-11-1 Nishikiori-Kita, Tondabayashi, Osaka \\ 584-8540, Japan: and ${ }^{d}$ Faculty of Science and Engineering, Setsunan University; 17-8 Ikeda-Nakamachi, Neyagawa, \\ Osaka 572-8508, Japan.
}

Received May 17, 2014; accepted June 17, 2014

The effects of administering the selenocompounds, sodium selenite, methylseleninic acid (MSA), and seleno-L-methionine (SeMet) on glucose tolerance were compared in the nicotinamide (NA) and streptozotocin (STZ)-induced diabetic mouse model. ICR mice were intraperitoneally treated twice with STZ (100 mg/ $\mathrm{kg}) 15 \mathrm{~min}$ after an injection of NA $(120 \mathrm{mg} / \mathrm{kg})$ at a 1-d interval. Non-fasting blood glucose levels were then monitored weekly while orally administering the selenocompounds at $158 \mu \mathrm{g} \mathrm{Se} / \mathrm{kg}$ body weight with free access to a selenium-deficient diet for 5 weeks. The mean body weights of NA/STZ-induced diabetic mice were partly restored by the administration of selenocompounds, while SeMet led to a higher selenium content and glutathione peroxidase 1 activity in the pancreas. Non-fasting and oral glucose tolerance-tested blood glucose levels, which were elevated by NA/STZ, were significantly suppressed by the administration of SeMet. These results suggest that SeMet may improve glucose tolerance in a NA/STZ-induced mild diabetic mouse model by increasing bioavailability in the pancreas.

Key words selenium; deficiency; diabetes; seleno-L-methionine; selenite; methylseleninic acid

Type 2 diabetes mellitus is a metabolic disease that develops as a result of defects in the secretion of insulin from pancreatic $\beta$-cells and actions of insulin on target tissues such as the liver, muscle, and adipose tissue. ${ }^{1,2)}$ This disease is characterized by a diabetes-specific microvascular pathology in the retina, renal glomerulus, and peripheral nervous system, and is the leading cause of blindness, end-stage renal disease, and neuropathy. ${ }^{3)}$ The development of this disorder has been linked to genetic and lifestyle factors, with obesity being the most common underlying factor. ${ }^{4,5}$ Even though type 2 diabetes is mostly preventable through lifestyle and diet modifications, ${ }^{6}$ its prevalence is expected to increase due to the worldwide obesity epidemic. ${ }^{7)}$ Such deteriorations in lifestyle have also been linked to oxidative stress in the pancreas. ${ }^{8)}$ The resulting enhancement in the production of reactive oxygen species (ROS) not only induces a dysfunction in pancreatic $\beta$-cells, but also inhibits insulin signal transduction and the actions of insulin, including increased glucose uptake ${ }^{9)}$ and glycogen synthesis. ${ }^{10)}$ However, development strategies to protect $\beta$-cells and insulin target tissues against oxidative stress may effectively prevent and treat type 2 diabetes. Therefore, an animal model needs to be established that is suitable for examining diabetes and its prevention. In the present study, we demonstrated that the ICR strain mouse was an appropriate animal model of nicotinamide (NA) and streptozotocin (STZ)-induced mild diabetes. ${ }^{11)}$

Selenium (Se) is an essential trace element for mammals and regulates many intracellular functions as a chemical component of selenoproteins. ${ }^{12)}$ The well-known selenoproteins are Se-dependent enzymes that include the glutathione (GSH) peroxidase (GPX) family and thioredoxin reductase (TR) family, which have selenocysteine residues in the catalytic centers and function as critical enzymes in response to oxidative stress. ${ }^{13,14)}$ As well as catalase (CAT), GPX1 eliminates hydrogen peroxide $\left(\mathrm{H}_{2} \mathrm{O}_{2}\right)$, which is intracellularly generated by oxidative stress or converted from superoxide anions by superoxide dismutase. ${ }^{15)}$ GPX1 is known to be the principal antioxidant enzyme for oxidative stress because CAT has low affinity for $\mathrm{H}_{2} \mathrm{O}_{2}{ }^{16}{ }^{16}$ Therefore, a Se deficiency may exacerbate the pathological conditions associated with diabetes by downregulating the expression of GPX1. Many epidemiological studies demonstrated that serum Se levels were lower in diabetic patients than in healthy individuals. ${ }^{17-19)}$ The atrophy and degeneration of pancreatic islets have also been reported in patients with the Se-deficiency syndrome, Keshan disease. ${ }^{20)}$ Se-insufficient rats were shown to have low serum insulin levels, which were normalized following supplementation with Se. ${ }^{21)}$ However, recent studies demonstrated that long-term and relatively high dietary supplementation with Se in U.S. adults increased the incidence of type 2 diabetes. ${ }^{22-24)}$ Therefore, it is debatable whether supplementation with Se is beneficial for preventing type 2 diabetes.

The objective of this study was to clarify an effective chemical form to supplement Se for the insufficient status and to compare Se bioavailability in pancreas and glucose tolerance with the administration of different selenocompounds: sodium selenite, methylseleninic acid (MSA), and seleno-L-methionine (SeMet), to an ICR mouse model with mild diabetes mellitus, which was developed by impairing insulin secretion with STZ and NA. These mice were fed a Se-deficient diet to prevent the influence of dietary Se sources. 


\section{MATERIALS AND METHODS}

Selenocompounds and Animals Sodium selenite (99.999\% purity), MSA (95\%), and SeMet ( $\geq 98 \%$ ) were purchased from Sigma-Aldrich Japan K.K. (Tokyo, Japan). Permission for the animal experiments performed in this study was obtained from the Animal Experiment Room Administration Committee of Setsunan University, and the experiments were conducted according to the Animal Experiment Guidelines of the Faculty of Pharmaceutical Sciences, Setsunan University. Specific pathogen-free male ICR mice (5 weeks old, about $25 \mathrm{~g}$ ) were purchased from Japan SLC Inc. (Shizuoka, Japan). The animals were maintained at $23 \pm 1{ }^{\circ} \mathrm{C}$, approximately $40 \%$ relative humidity and a light/dark cycle of $12 \mathrm{~h}$ each, and had ad libitum access to $\gamma$-ray-irradiated pelleted rodent chow (Type NMF, Oriental Yeast Co., Tokyo, Japan) and tap water. The mice were acclimated for 1 week prior to use.

NA/STZ Treatment and Selenocompound Administration The ICR mice (10 mice/group) were fasted for $16 \mathrm{~h}$ and then intraperitoneally injected with NA (Sigma-Aldrich) dissolved in saline at a dose of $120 \mathrm{mg} / \mathrm{kg}$ body weight. Fifteen minutes after the NA treatment, the mice were intraperitoneally injected with STZ (Sigma-Aldrich) freshly dissolved in $50 \mathrm{mmol} / \mathrm{L}$ citrate buffer ( $\mathrm{pH} 4.5$ ) at a dose of $100 \mathrm{mg} / \mathrm{kg}$ body weight. After an interval of $1 \mathrm{~d}$, NA and STZ were administered again in the same manner. The next day, animals were orally administered a selenocompound dissolved in saline at a dose of $158 \mu \mathrm{g}$ Se $(2 \mu \mathrm{mol}) / \mathrm{kg}$ body weight $/ \mathrm{d}$ using a stainless steel gavage needle and a $1 \mathrm{~mL}$-syringe and had ad libitum access to sterilized water and a $\gamma$-ray-irradiated torula yeast (KR yeast; Kohjin Life Sciences Co., Tokyo, Japan)-based diet (30.0\% yeast, $17.0 \% \alpha$-cornstarch, 23.7\% $\beta$-cornstarch, $10.0 \%$ sucrose, $13.0 \%$ lard, $5.0 \%$ mineral mix, $0.85 \%$ vitamin mix, $0.3 \%$ DL-methionine, $0.15 \%$ choline chloride; Oriental Yeast Co., Tokyo, Japan) containing $32.3 \pm 2.92 \mathrm{ng} \mathrm{Se} / \mathrm{g}$ for 5 weeks, while monitoring body weight. The animal experiments described in this study were performed separately for each selenocompound including the control and NA/STZ-alone groups.

Assays of Blood Glucose, Tissue Se, and GPX1 Non- fasting blood glucose levels were measured before and after the treatment with NA/STZ and monitored weekly for 5 weeks. Blood was drawn from the tail vein at 10:00-12:00. The oral glucose tolerance test (OGTT) was performed orally administering glucose at a dose of $2000 \mathrm{mg} / \mathrm{kg}$ body weight to overnight-fasted mice 5 weeks after the treatment with NA/ STZ. Blood was collected from the tail vein 0, 30, 60, 90, and $120 \mathrm{~min}$ after glucose loading. Glucose levels in these blood samples were determined using Glucose Pilot (Aventir Biotech, LLC, West Carlsbad, CA, U.S.A.). After OGTT, the liver was perfused with ice-cold saline through the portal vein under pentobarbital anesthesia, and the liver and pancreas were then removed and immediately stored in $-80^{\circ} \mathrm{C}$ for storage until assayed. After wet digestion of the tissues (the pancreases of 2 mice were randomly mixed within the same treatment group to increase the quantity) with a mixed acid solution of nitric acid-perchloric acid $(2: 1, \mathrm{v} / \mathrm{v})$, the Se content was determined by the fluorometric method using 2,3-diaminonaphthalene. ${ }^{25)}$

Pancreatic GPX1 activity was determined by the method described by Lawrence and Burk ${ }^{26)}$ with slight modifications. ${ }^{27)}$ The pancreas was homogenized with 20 vol. ice-cold $0.2 \%$ Triton-X-50 mmol/L phosphate buffer $(\mathrm{pH} 7.0)$ and the homogenate was centrifuged at $13000 \times \boldsymbol{g}$ for $15 \mathrm{~min}$ at $4^{\circ} \mathrm{C}$. The supernatant was dialyzed with the 8000 molecular weight (MW)-cut-off membrane against $50 \mathrm{mmol} / \mathrm{L}$ phosphate buffer ( $\mathrm{pH}$ 7.0) to remove glucose metabolites such as methylglyoxal, which can react with $\mathrm{H}_{2} \mathrm{O}_{2}$ added as the substrate. The reaction mixture consisted of $50 \mathrm{mmol} / \mathrm{L}$ phosphate buffer $(\mathrm{pH}$ 7.0), $0.2 \mathrm{mmol} / \mathrm{L}$ nicotinamide adenine dinucleotide phosphate (NADPH), $2 \mathrm{mmol} / \mathrm{L}$ GSH, $0.2 \mathrm{mmol} / \mathrm{L}$ sodium azide, and 1 unit/mL GSH reductase in a total volume of $850 \mu \mathrm{L}$. The dialyzed pancreas supernatant $(100 \mu \mathrm{L})$ was added to the reaction mixture and incubated for $2 \mathrm{~min}$ at $25^{\circ} \mathrm{C}$ before initiating the reaction by the addition of $50 \mu \mathrm{L} 0.3 \mathrm{mmol} / \mathrm{L} \mathrm{H}_{2} \mathrm{O}_{2}$ solutions. Absorbance at $340 \mathrm{~nm}$ was recorded for $1 \mathrm{~min}$ and the activity was calculated from the slope of these lines as nanomoles NADPH oxidized per min. Protein was determined by the Bradford method ${ }^{28)}$ using bovine serum albumin as the protein
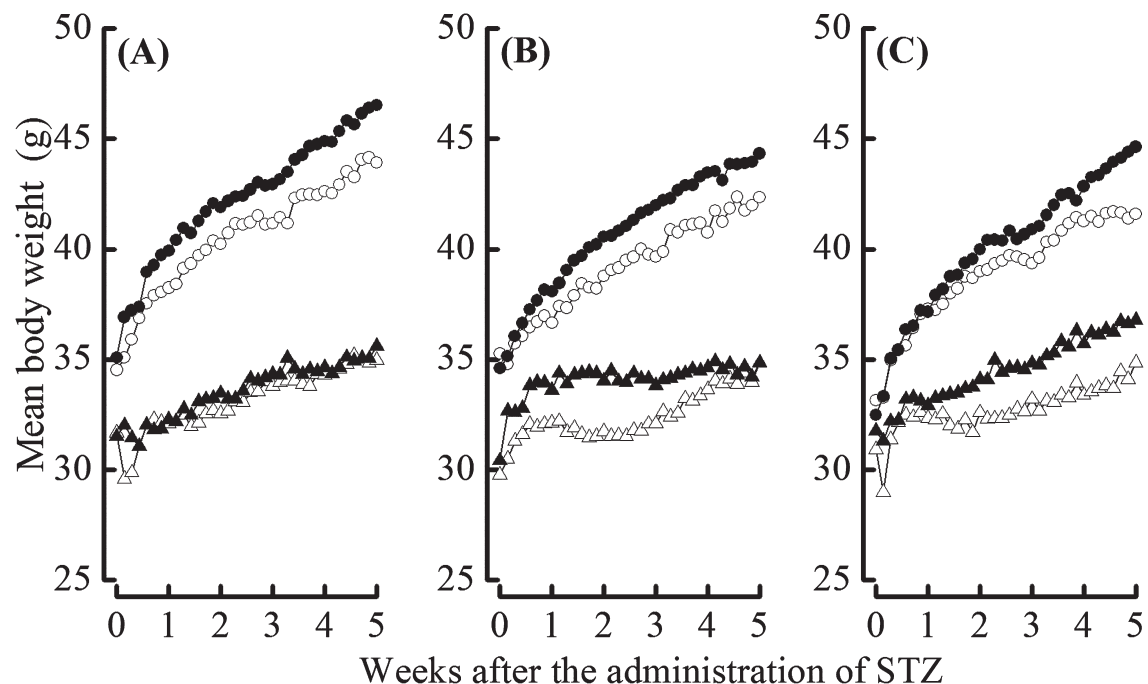

Weeks after the administration of STZ

Fig. 1. Effects of Administering Sodium Selenite (A), MSA (B), and SeMet (C) on Body Weight

Control $(\bigcirc)$, selenocompound administration $(\bullet)$, NA/STZ treatment $(\triangle)$ and NA/STZ treatment+selenocompound administration $(\Delta)$. The values shown are the mean \pm S.D. $(n=10)$. 
standard.

Statistical Analysis Values in the figures are expressed as the means \pm S.D. Statistical analysis was carried out by a oneway ANOVA with a Bonferroni correction. The $p$ level was set at 0.05 or 0.01 .

\section{RESULTS}

Pancreatic Se Bioavailability of Selenocompounds The effects of administering the different selenocompounds on body weight gain, hepatic and pancreatic Se contents, and pancreatic GPX1 activity in NA/STZ-induced diabetic mice were investigated to compare their Se availabilities. All 3 selenocompounds augmented the mean body weights in NA/STZuntreated mice (Fig. 1). Body weight gain was suppressed by the NA/STZ treatment. However, body weight was partly restored by the supplementation with SeMet for 5 weeks (Fig. 1C). Both hepatic and pancreatic Se contents significantly increased due to supplementation with the selenocompounds regardless of the NA/STZ treatment, and were the highest with the SeMet administration (Fig. 2). Pancreatic GPX1 activity was significantly enhanced by SeMet, whereas the NA/STZ treatment suppressed its activity (Fig. 3).

Glucose Tolerance by Supplementary Selenocompounds To investigate the effects of administering the different selenocompounds on glucose tolerance in NA/STZ-induced diabetic mice, non-fasting blood glucose levels before and 1-5 weeks after the NA/STZ treatment as well as glucose levels during OGTT after the 5 weeks were monitored. Supplementation with the selenocompounds had no significant influence on

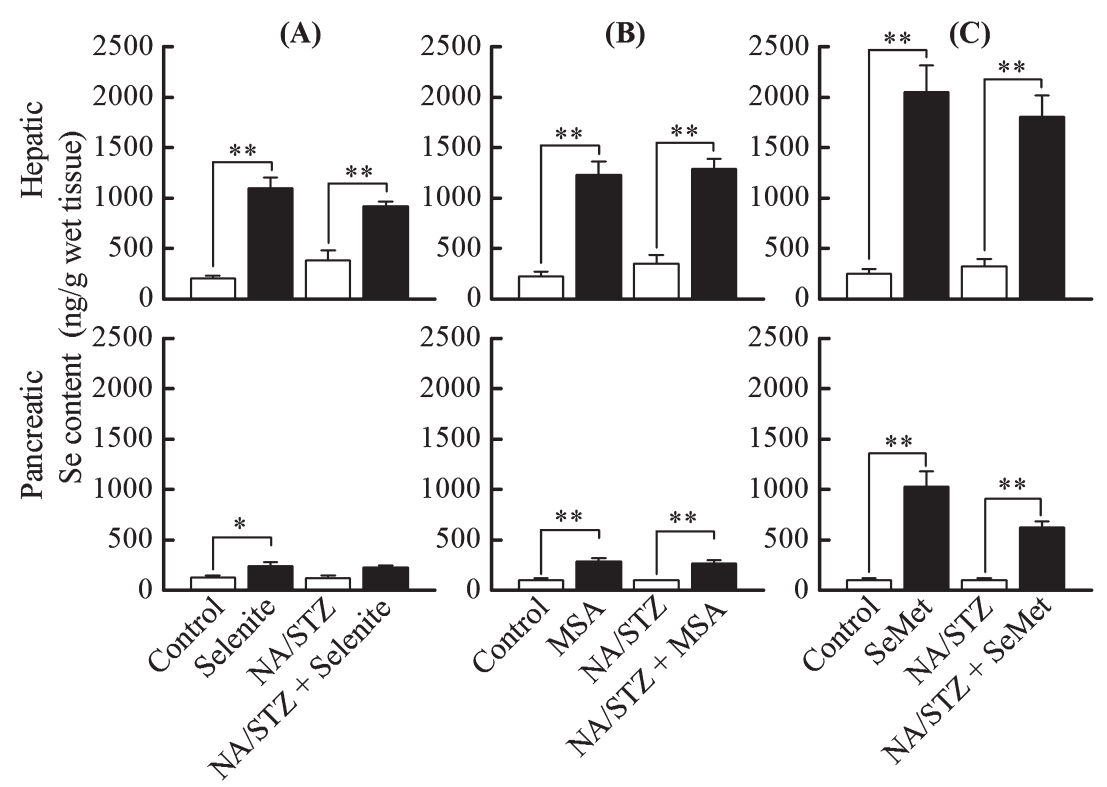

Fig. 2. Effects of Administering Sodium Selenite (A), MSA (B), and SeMet (C) on Hepatic and Pancreatic Se Contents The values shown are the mean \pm S.D. $(n=10)$. Significantly different from the control or NA/STZ treatment group at $* p<0.05 ; * * p<0.01$.
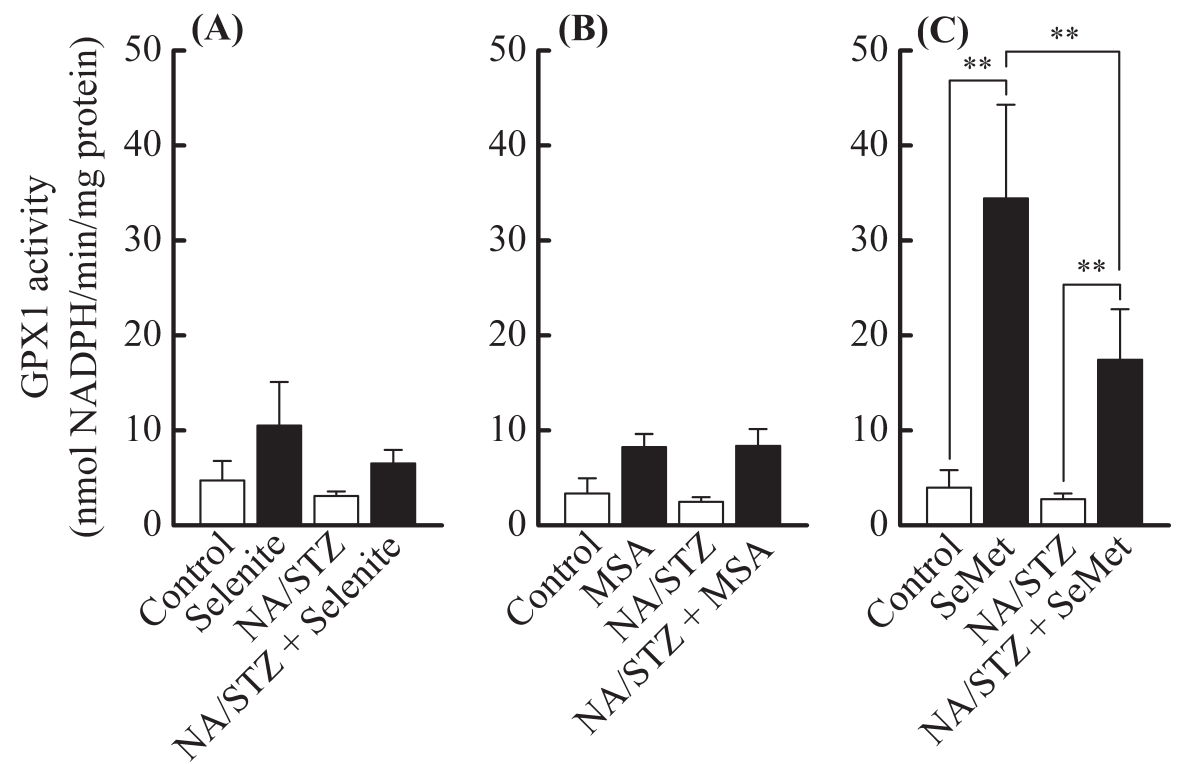

Fig. 3. Effects of Administering Sodium Selenite (A), MSA (B), and SeMet (C) on Pancreatic GPX1 Activity

The values shown are the mean \pm S.D. $(n=10)$. Significantly different from the control or NA/STZ treatment group at $* * p<0.01$. 

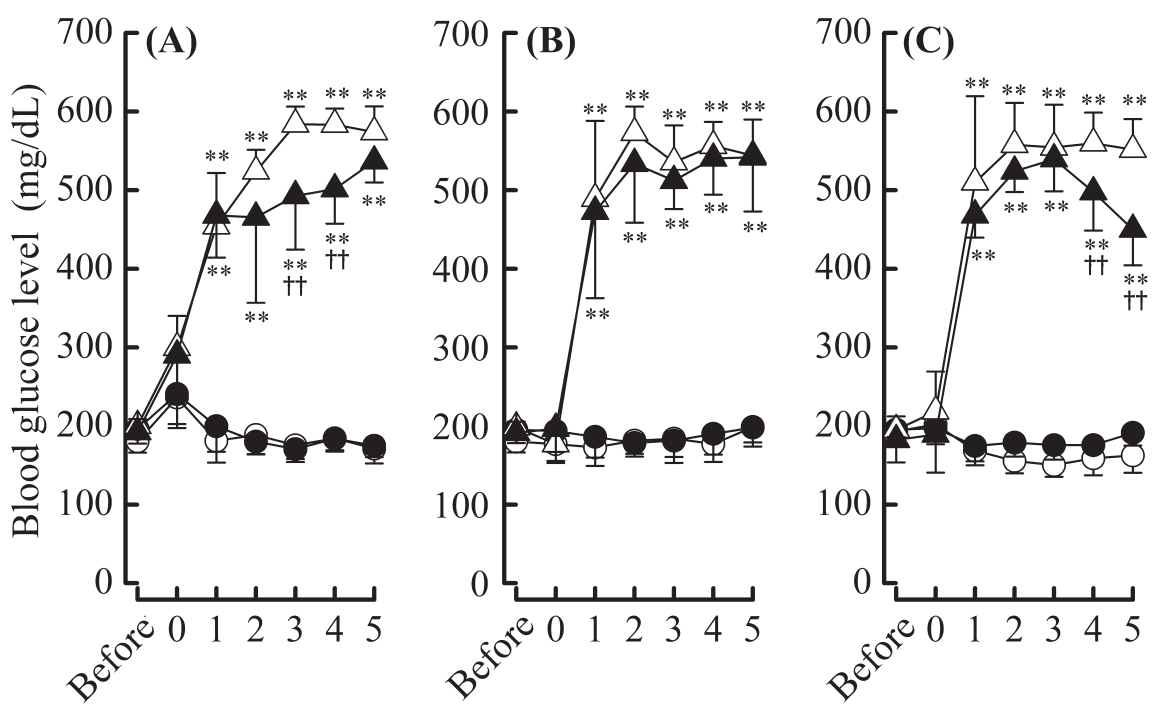

Weeks after the administration of STZ

Fig. 4. Effects of Administering Sodium Selenite (A), MSA (B), and SeMet (C) on Non-fasting Blood Glucose Levels

Non-fasting blood glucose levels were measured before and after the treatment with NA/STZ and monitored weekly for 5 weeks. Control (O), selenocompound administration $(\bullet)$, NA/STZ treatment $(\triangle)$ and NA/STZ treatment+selenocompound administration $(\mathbf{\Lambda})$. The values shown are the mean \pm S.D. $(n=10)$. Significantly different from the control group at $* * p<0.01$ and from the NA/STZ treatment group at ${ }^{\dagger \dagger} p<0.01$.

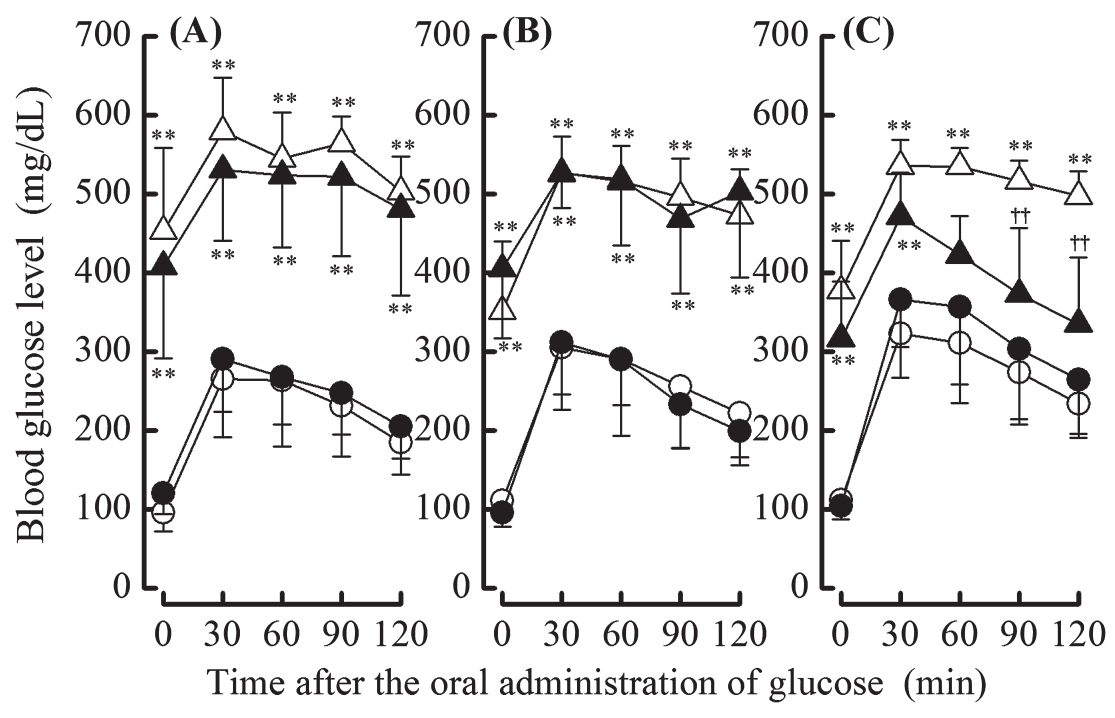

Fig. 5. Effects of Administering Sodium Selenite (A), MSA (B), and SeMet (C) on Blood Glucose Levels during OGTT

OGTT was performed by orally administering glucose at a dose of $2000 \mathrm{mg} / \mathrm{kg}$ body weight to overnight-fasted mice 5 weeks after the treatment with NA/STZ. Control $(\mathrm{O})$, selenocompound administration $(\mathbf{\bullet})$, NA/STZ treatment $(\triangle)$ and NA/STZ treatment+selenocompound administration $(\mathbf{\Lambda})$. The values shown are the mean \pm S.D. $(n=10)$. Significantly different from the control group at ${ }^{* *} p<0.01$ and from the NA/STZ treatment group at ${ }^{1 \dagger} p<0.01$.

non-fasting glucose levels in NA/STZ-untreated mice (Fig. 4). However, selenite and SeMet significantly suppressed the nonfasting blood glucose levels that were increased by the NA/ STZ treatment after 3-4 weeks and 4-5 weeks, respectively (Figs. 4A,C). Although blood glucose levels during OGTT increased in NA/STZ-treated mice, only the administration of SeMet significantly lowered glucose levels 90-120 min after glucose loading (Fig. 5).

\section{DISCUSSION}

Type 2 diabetes is characterized by hyperglycemia in the context of insulin resistance, which precedes an insulin deficiency by pancreatic $\beta$-cell failure as a response to oxidative stress, endoplasmic reticulum stress, and thereby inflamma- tion. $^{29)}$ STZ is an agent that acts as a nitric oxide donor and is frequently used to induce experimental diabetes in mammals. ${ }^{30}$ This chemical specifically produces free radicals in $\beta$-cells and causes the fragmentation of DNA chains, which evokes the activation of poly(ADP-ribose)-polymerase and consumption of a large amount of intracellular NAD to repair damaged DNA. ${ }^{30)}$ The intake of NA has been shown to effectively supplement the consumption of NAD, ${ }^{31)}$ which ultimately protects $\beta$-cells against the disorder induced by the STZ treatment. In our previous study, the induction of experimental diabetes by the NA/STZ-treatment in ICR strain mice was considered to be restricted more than with the STZ treatment alone and was sensitive to hyperglycemia under diabetic conditions. ${ }^{11)}$ Using the NA/STZ-induced mild diabetic mouse model, the objective of this study was to compare Se bioavail- 
ability in pancreas and glucose tolerance with the administration of different selenocompounds: sodium selenite, MSA, and SeMet. Considering the biological availability of Se, it is necessary for Se sources to be metabolized to hydrogen selenide $\left(\mathrm{H}_{2} \mathrm{Se}\right)$, which is a physiologically active form that is incorporated into the selenocysteine residues of selenoenzymes. ${ }^{32)}$ Selenite can be reduced to $\mathrm{H}_{2} \mathrm{Se}$ by 2 molecules of GSH and the reductase. ${ }^{33)}$ MSA can be converted by 4 molecules of GSH to methylselenol, ${ }^{34)}$ which can be reversibly converted to $\mathrm{H}_{2} \mathrm{Se}^{35}$ ) SeMet can be directly converted to methylselenol by cystathionine $\gamma$-lyase ${ }^{36)}$ as well as $\mathrm{H}_{2} \mathrm{Se}$ through the L-methionine metabolic pathway and seleno-L-cysteine $\beta$-lyase. ${ }^{37)}$

To avoid the effects of internal Se sources as previously described, ${ }^{38)}$ mice were fed a Se-deficient diet for 5 weeks in the present study. We then compared the effects of these selenocompounds as agents for $\mathrm{H}_{2} \mathrm{Se}$ and/or methylselenol metabolic pools on body weight gain, hepatic and pancreatic Se contents, pancreatic GPX1 activity, and blood glucose levels in NA/ STZ-induced diabetic mice. The dosage of $158 \mu \mathrm{g}$ Se $(2 \mu \mathrm{mol}) /$ $\mathrm{kg}$ body weight/d was decided as an intake within the physiological range of mouse, which was estimated from Se level in the normal diet and the dietary intake. ${ }^{27}$ The administration of the selenocompounds had no detrimental effects on the appearance of the coat, general grooming, or non-fasting glucose levels in NA/STZ-untreated mice. As shown in Fig. 1 , the difference observed in mean body weight between the selenocompound-administered and control groups reflected the influence of a Se deficiency. As shown in Figs. 2 and 3, SeMet exhibited the highest bioavailability of the 3 selenocompounds examined, particularly in the pancreatic Se content and GPX1 activity. Only SeMet-supplemented mice exceeded the normal diet-feeding mice in the Se content and GPX1 activity of NA/ STZ-untreated groups, as compared with NSY mice that were established as an inbred strain with spontaneous development of diabetes from ICR mice. ${ }^{27)}$ As cystathionine $\gamma$-lyase activity is known to be relatively high in the liver and pancreas, ${ }^{39}$ ) methylselenol may easily be formed from SeMet in these tissues. In our previous study, the activity of GPX4 and TR in the pancreas was negligible. ${ }^{27)}$ Therefore, GPX1 may be important in the defense system against oxidative stress. As shown in Figs. 4 and 5, SeMet significantly suppressed nonfasting and OGTT glucose levels in NA/STZ-treated mice. However, the administration of MSA had no effect on glucose levels, whereas selenite slightly decreased non-fasting glucose levels. These results suggest that the Se agent for GSHdependent $\mathrm{H}_{2} \mathrm{Se}$ or methylselenol metabolic pool shows low $\mathrm{Se}$ bioavailability and may not contribute to an improvement in diabetes. Chronic hyperglycemia decreases the expression of pancreatic $\gamma$-glutamylcysteine ligase, which is the rate-limiting enzyme for GSH synthesis. ${ }^{40)}$ A Se deficiency was also shown to decrease the pancreatic GSH content. ${ }^{27)}$ Selenite and MSA may consume GSH to form $\mathrm{H}_{2} \mathrm{Se}$ or methylselenol in the pancreas, which has a low Se content. Taken together, a Se agent that has $\mathrm{GSH}$-independent $\mathrm{H}_{2} \mathrm{Se}$ or a methylselenol metabolic pool may be suitable for the primary prevention of diabetes.

In conclusion, SeMet improved glucose tolerance in the NA/STZ-induced mild diabetic mouse model by exhibiting high bioavailability in the pancreas. In this study, there were no significant differences in non-fasting plasma insulin levels between the control and the selenocompound-administration groups because their levels were very low by NA/STZ treat- ment and close to the detection limit of ELISA assay (data not shown). Further studies will be needed to clarify the protection mechanisms of SeMet from hyperglycemia and an insulin deficiency including assessments of hemoglobin A1c and OGTT plasma insulin.

Acknowledgments This work was supported in part by a Grant-in-Aid for Scientific Research (KAKENHI 20590128) from the Japan Society for the Promotion of Science.

\section{REFERENCES}

1) Bell GI, Polonsky KS. Diabetes mellitus and genetically programmed defects in $\beta$-cell function. Nature, 414, 788-791 (2001).

2) Das SK, Chakrabarti R. Non-insulin dependent diabetes mellitus: present therapies and new drug targets. Mini Rev. Med. Chem., 5, 1019-1034 (2005).

3) Brownlee M. Biochemistry and molecular cell biology of diabetic complications. Nature, 414, 813-820 (2001).

4) Zimmet P, Alberti KG, Shaw J. Global and societal implications of the diabetes epidemic. Nature, 414, $782-787$ (2001).

5) Narayan KMV, Kanaya AM, Gregg EW. Lifestyle intervention for the prevention of type 2 diabetes mellitus: putting theory to practice. Treat. Endocrinol., 2, 315-320 (2003).

6) $\mathrm{Hu}$ FB. Globalization of diabetes: The role of diet, lifestyle, and genes. Diabetes Care, 34, 1249-1257 (2011).

7) Whiting DR, Guariguata L, Weil C, Shaw J. IDF diabetes atlas: global estimates of the prevalence of diabetes for 2011 and 2030. Diabetes Res. Clin. Pract., 94, 311-321 (2011).

8) Evans JL, Goldfine ID, Maddux BA, Grodsky GM. Oxidative stress and stress-activated signaling pathways: a unifying hypothesis of type 2 diabetes. Endocr. Rev., 23, 599-622 (2002).

9) D’Apolito M, Du X, Zong H, Catucci A, Maiuri L, Trivisano T, Pettoello-Mantovani M, Campanozzi A, Raia V, Pessin JE, Brownlee M, Giardino I. Urea-induced ROS generation causes insulin resistance in mice with chronic renal failure. J. Clin. Invest., 120, 203-213 (2010).

10) Bloch-Damti A, Bashan N. Proposed mechanisms for the induction of insulin resistance by oxidative stress. Antioxid. Redox Signal., 7, 1553-1567 (2005).

11) Shimizu R, Sakazaki F, Okuno T, Nakamuro K, Ueno H. Difference in glucose intolerance between C57BL/6J and ICR strain mice with streptozotocin/nicotinamide-induced diabetes. Biomed. Res., 33, 63-66 (2012).

12) Allan CB, Lacourciere GM, Stadtman TC. Responsiveness of selenoproteins to dietary selenium. Annu. Rev. Nutr., 19, 1-16 (1999).

13) Arnér ESJ, Holmgren A. Physiological functions of thioredoxin and thioredoxin reductase. Eur. J. Biochem., 267, 6102-6109 (2000).

14) Patrick L. Selenium biochemistry and cancer: a review of the literature. Altern. Med. Rev., 9, 239-258 (2004).

15) Yan H, Harding JJ. Glycation-induced inactivation and loss of antigenicity of catalase and superoxide dismutase. Biochem. J., 328, 599-605 (1997).

16) Matés JM, Sánchez-Jiménez F. Antioxidant enzymes and their implications in pathophysiologic processes. Front. Biosci., 4, D339D345 (1999).

17) Tan M, Sheng L, Qian Y, Ge Y, Wang Y, Zhang H, Jiang M, Zhang $\mathrm{G}$. Change of serum selenium in pregnant women with gestational diabetes mellitus. Biol. Trace Elem. Res., 83, 231-237 (2001).

18) Kornhauser C, Garcia-Ramirez JR, Wrobel K, Pérez-Luque EL, Garay-Sevilla ME, Wrobel K. Serum selenium and glutathione peroxidase concentrations in type 2 diabetes mellitus patients. Prim. Care Diabetes, 2, 81-85 (2008).

19) Molnar J, Garamvolgyi Z, Herold M, Adanyi N, Somogyi A, Rigo J Jr. Serum selenium concentrations correlate significantly with 
inflammatory biomarker high-sensitive CRP levels in Hungarian gestational diabetic and healthy pregnant women at mid-pregnancy. Biol. Trace Elem. Res., 121, 16-22 (2008).

20) Köhrle J, Jakob F, Contempre B, Dumont JE. Selenium, the thyroid, and the endocrine system. Endocr. Rev., 26, 944-984 (2005).

21) Tong WM, Wang F. Alterations in rat pancreatic islet beta cells induced by Keshan disease pathogenic factors: protective action of selenium and vitamin E. Metabolism, 47, 415-419 (1998).

22) Bleys J, Navas-Acien A, Guallar E. Selenium and diabetes: more bad news for supplements. Ann. Intern. Med., 147, 271-272 (2007).

23) Bleys J, Navas-Acien A, Guallar E. Serum selenium and diabetes in U.S. dults. Diabetes Care, 30, 829-834 (2007).

24) Stranges S, Marshall JR, Natarajan R, Donahue RP, Trevisan M, Combs GF, Cappuccio FP, Ceriello A, Reid ME. Effects of longterm selenium supplementation on the incidence of type 2 diabetes. Ann. Intern. Med., 147, 217-223 (2007).

25) Walkinson JH. Fluorometric determination of selenium in biological material with 2,3-diaminonaphthalene. Anal. Chem., 38, 92-97 (1966).

26) Lawrence RA, Burk RF. Glutathione peroxidase activity in selenium-deficient rat liver. Biochem. Biophys. Res. Commun., 71 952-958 (1976)

27) Shimizu R, Ueno H, Okuno T, Sakazaki F, Nakamuro K. Effect of sodium selenite supplementation on glucose intolerance and pancreatic oxidative stress in type 2 diabetic mice under different selenium status. J. Health Sci., 55, 271-280 (2009).

28) Bradford MM. A rapid and sensitive method for the quantitation of microgram quantities of protein utilizing the principle of proteindye binding. Anal. Biochem., 72, 248-254 (1976).

29) Montane J, Cadavez L, Novials A. Stress and the inflammatory process: a major cause of pancreatic cell death in type 2 diabetes. Diabetes Metab. Syndr. Obes., 7, 25-34 (2014).

30) Szkudelski T. The mechanism of alloxan and streptozotocin action in B cells of the rat pancreas. Physiol. Res., 50, 537-546 (2001).

31) Uchigata $Y$, Yamamoto $H$, Kawamura A, Okamoto H. Protection by superoxide dismutase, catalase, and poly(ADP-ribose) synthetase inhibitors against alloxan- and streptozotocin-induced islet DNA strand breaks and against the inhibition of proinsulin synthesis. $J$. Biol. Chem., 257, 6084-6088 (1982).

32) Mizutani T, Kurata H, Yamada K. Study of mammalian selenocysteyl-tRNA synthesis with [75Se]HSe. FEBS Lett., 289, 59-63 (1991).

33) Suzuki KT, Ogra Y. Metabolic pathway for selenium in the body: speciation by HPLC-ICP MS with enriched Se. Food Addit. Contam., 19, 974-983 (2002).

34) Sinha R, Unni E, Ganther HE, Medina D. Methylseleninic acid, a potent growth inhibitor of synchronized mouse mammary epithelial tumor cells in vitro. Biochem. Pharmacol., 61, 311-317 (2001).

35) Suzuki KT, Tsuji Y, Ohta Y, Suzuki N. Preferential organ distribution of methylselenol source Se-methylselenocysteine relative to methylseleninic acid. Toxicol. Appl. Pharmacol., 227, 76-83 (2008).

36) Okuno $T$, Ueno $H$, Nakamuro K. Cystathionine gamma-lyase contributes to selenomethionine detoxification and cytosolic glutathione peroxidase biosynthesis in mouse liver. Biol. Trace Elem. Res., 109, 155-171 (2006)

37) Soda K, Oikawa T, Esaki N. Vitamin B6 enzymes participating in selenium amino acid metabolism. Biofactors, 10, 257-262 (1999).

38) Ueno H, Hasegawa G, Ido R, Okuno T, Nakamuro K. Effects of selenium status and supplementary seleno-chemical sources on mouse T-cell mitogenesis. J. Trace Elem. Med. Biol., 22, 9-16 (2008).

39) Okuno T, Motobayashi S, Ueno H, Nakamuro K. Purification and characterization of mouse hepatic enzyme that converts selenomethionine to methylselenol by its alpha, gamma-elimination. Biol. Trace Elem. Res., 106, 77-94 (2005).

40) Robertson RP. Chronic oxidative stress as a central mechanism for glucose toxicity in pancreatic islet beta cells in diabetes. J. Biol. Chem., 279, 42351-42354 (2004). 